\title{
3D electroplated microstructures fabricated by a novel height control method
}

\author{
L.-W. Pan, P. Yuen, L. Lin
}

\begin{abstract}
A 3D electroplating process by means of pixelwise, step height control of selective electroplating has been successfully demonstrated. A total of eight rectangular pixels of $80 \times 20 \mu \mathrm{m}^{2}$ in area and $1.1-9.3 \mu \mathrm{m}$ in height have been fabricated with a height difference of $1 \mu \mathrm{m}$ in adjacent pixels. The process requires only two masks in a single electroplating process. Nickel electroplating is selected as the demonstrating material and is performed at $50{ }^{\circ} \mathrm{C}$ with current density of $400 \mathrm{~A} / \mathrm{m}^{2}$ for $30 \mathrm{~min}$. A theoretical model in electroplating process is discussed to assist the design of 3D microstructures and verified with the experimental data. As such, this process has potential applications in making pixel-wise, 3D microstructures with precise height control.
\end{abstract}

\section{1}

\section{Introduction}

High-aspect-ratio microstructures fabricated by electroplating process, such as LIGA [1], has been generally considered as 2.5-D devices. One limiting factor is that the deposition thickness from electroplating is the same for the height of microstructures and thus constraining the $3 \mathrm{D}$ manufacturing capability. Although EFAB (Electrochemical Fabrication) [2], Stereolithography [3] and laser Stereolithography processes [4] have been proposed to fabricate $3 \mathrm{D}$ microstructures, they require repetitive alignment and deposition procedures in a layer-by-layer manufacturing scheme. This work presents a novel and yet

Received: 10 August 2001/Accepted: 24 September 2001

L.-W. Pan ( $\bowtie)$

Berkeley Sensor and Actuator Center, Mechanical Engineering,

The University of Michigan, 1113 Etchevery Hall, Berkeley, CA 94720-1740, USA

e-mail: lwpan@me.berkeley.edu

P. Yuen, L. Lin

Berkeley Sensor and Actuator Center, Mechanical Engineering,

University of California at Berkeley, USA

The authors would like to thank Mr. R. Wilson for taking SEM pictures. This project is supported in part by a NSF award (DMI-0096220) and a DARPAR award under the MTO/MEMS program.

This paper was presented at the Fourth International Workshop on High Aspect Ratio Microstructure Technology HARMST 2001 in June 2001. simple approach to control the step-height for 3D microstructures in a single electroplating process. A theoretical model is developed in order to characterize the process and help design of 3D microstructures.

\section{2}

\section{Working principle}

Figure 1 shows the schematic of the new approach where only eight pixels are constructed in this demonstration experiment. First, 500/2000 ̊ thick of Cr/Au pixels with $80 \mu \mathrm{m}$ in length and $20 \mu \mathrm{m}$ in width are evaporated and patterned by the lift-off process on a silicon substrate that has a layer of $0.8 \mu \mathrm{m}$ silicon dioxide on top. Afterwards, a $9 \mu \mathrm{m}$ thick photoresist is coated and patterned to open the designated areas for electroplating. Nickel electroplating is performed at $50{ }^{\circ} \mathrm{C}$ with current density of $400 \mathrm{~A} / \mathrm{m}^{2}$ for $30 \mathrm{~min}$. At completion, the deposition thickness on the eight pixels varies as controlled by the step-height control electrodes.

Figure 2 shows the control mechanism. The common electrode is connected to an external current supply. The gaps between the common electrode and the designated electroplating pixels increases from 0 (No. 0 pixel) to $7 \mu \mathrm{m}$ (No. 7 pixel) with an increment amount of $1 \mu \mathrm{m}$ between adjacent pixels. These gaps serve as controllers to initiate the deposition process on the target pixels. When the lateral deposition on the common electrode fills the control gap, the target electrode becomes active. A $1 \mathrm{~cm}^{2}$ open metal area is designed to be exposed to the electrolyte in addition to the microstructure deposition area as a current reservoir to prevent the fluctuation of the current density. Figure 3 shows step-height control electrodes and the common electrode with patterned photoresist. It can be clearly observed that the first and second step-height control electrodes are connected to the common electrode due to the defective lift-off process.

3

\section{Discussions}

The growth of each individual pixel is activated by the lateral growth of electroplated nickel and the deposition rate is affected by various parameters including mass transport, electron transfer, electrical potential, chemical potential, and crystal growth. In the nickel deposition process, a nickel ion must reach the electrode/solution interface, obtain electrons and be reduced to a nickel atom. The crystal nickel structure [5] is formed when multiple nickel atoms are piled up. Various effects are discussed for better understanding of the height control process as proposed in this work. 


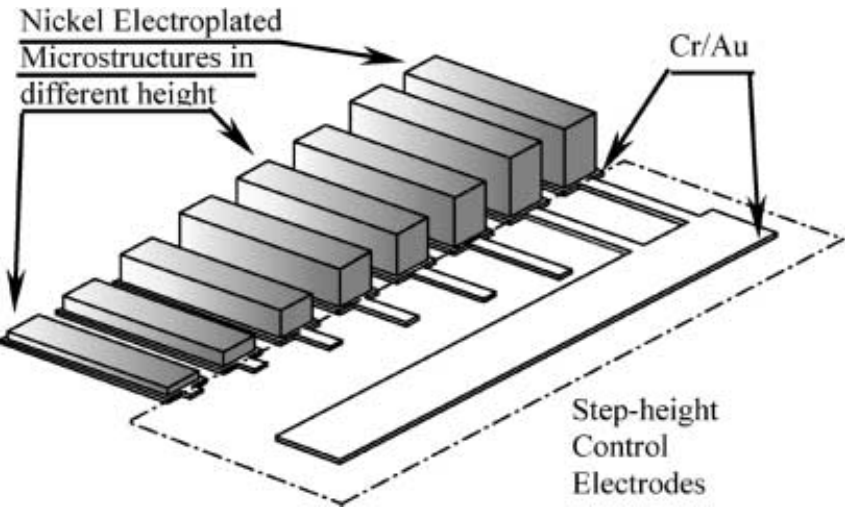

Fig. 1. The schematic drawing of 3D microstructures fabricated by the step-height control method

\section{1}

\section{Electron Transfer}

At the electrode/solution interface, chemical and electrical potential reaches equilibrium and forms a 1-30 nm thick charged region known as the double layer $[6,7]$. An activation energy is required for an ion or molecule to travel through the double layer for electron transfer and an equal energy is required for an atom or a molecule leaving the surface. If the electrical potential is changed, a new equilibrium state is expected. In most electroplating processes, the electron transfer is easily accomplished as compared with the mass transport such that mass transfer dominates the deposition rate of the electroplating process.

\section{2}

\section{Mass transport}

The primary mechanisms for mass transport of metal ions from anode to cathode for deposition are ion migration, convection and diffusion processes driven by electrical potential, pressure, and concentration gradient, respectively.

\subsection{1}

\section{Electric field induced ion migration}

Charged spices in electrolyte can be driven by the electrical potential. The current density resulted from the migration of charged species is proportional to the electric field and conductivity of the electrolyte as described by Ohm's law [6]. In a conventional electroforming process like the one presented here, the electric field is weak because the cathode and anode are placed far away in a beaker. Therefore, ion migration due to electric field only plays a secondary role in the mass transport process and is ignored in this paper.

\subsection{2}

\section{Ion transport due to the convection}

The consumption of nickel ions around the electrode surface creates the natural convection as the result of density gradient. Agitation is often used to enhance the convection process. In this paper, a $2.54-\mathrm{cm}$ long magnetic stirring bar rotating at $250 \mathrm{rpm}$ is used in a beaker of $12.7 \mathrm{~cm}$ in diameter. The stirring bar creates an electrolyte flow with an estimated speed of $0.15 \mathrm{~m} / \mathrm{s}$ and the boundary

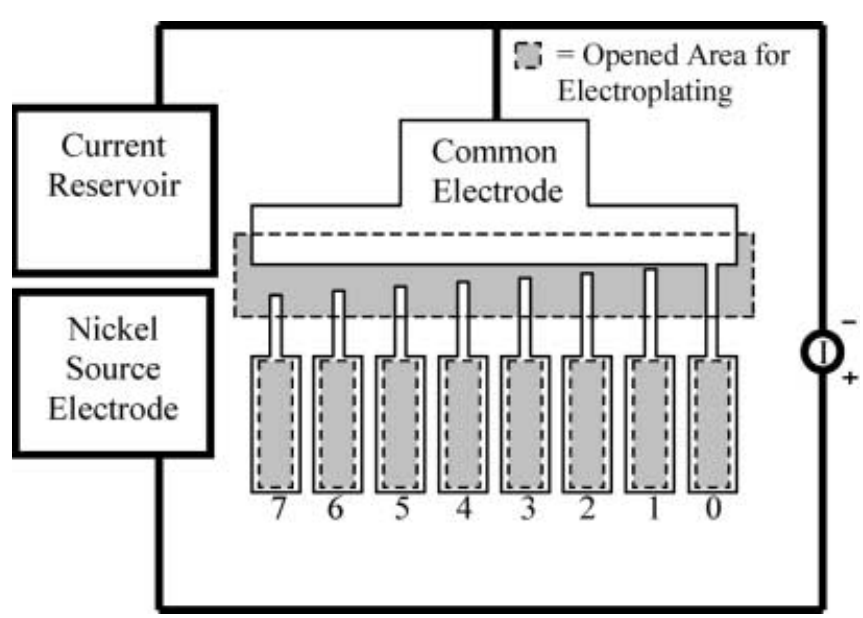

Fig. 2. The illustration of the control mechanism for the $3 \mathrm{D}$ Electroplating process

layer, $\delta$, on the surface of $1 \times 1 \mathrm{~cm}$ die is estimated by Blasius equation [8].

$\frac{\delta}{L}=\frac{5.0}{\operatorname{Re}_{L}^{1 / 2}}$

where

$\operatorname{Re}_{L}=\frac{V L}{v}$

The boundary layer is calculated as $900 \mu \mathrm{m}$ as shown in Fig. 4 by applying the characteristic length as $L=0.01 \mathrm{~m}$, and $v \approx 5.5 \times 10^{-7} \mathrm{~m}^{2} / \mathrm{s}$, the kinetic viscosity of water as the electrolyte kinetic viscosity.

\subsection{3}

\section{Ion transport due to diffusion}

There are two mechanisms, current limit deposition and diffusion limit deposition, determining the pixel height deposition rate. The current limit deposition is valid if the current density is smaller than the diffusion current density and the deposition rate is proportional to the input current density. When the input current is increased and saturated at the diffusion limit current, the deposition rate is decided by the nickel ion diffusion flux. Forced convection (to reduce the thickness of boundary layer) and rising the processing temperature (to increase the mobility of nickel ions) are possible methods to increase the ion transport process.

Diffusion happens in the boundary layer. With the existence of forced convection, the diffusion current density, $j_{\text {diff }}$, is proposed as [9]:

$j_{\text {diff }}=\frac{n F D_{0} C_{0}}{\delta}$

where $D_{0}$ is the diffusion coefficient of nickel ion. The bulk concentration of electrolyte is represented as $C_{0}$ and is approximated as $0.8 \times 10^{3} \mathrm{~mol} / \mathrm{m}^{3}$ [10]. $F$ is Faraday $\left(9.6485 \times 10^{4} \mathrm{C} / \mathrm{mol}\right)$. The number of electron reduced or oxidized per molecule is represented as $\mathrm{n}$ and is $2\left(\mathrm{Ni}^{2+}\right)$ in this case. The diffusion current density is measured at $400 \mathrm{~A} / \mathrm{m}^{2}$ by monitoring the current changes in the electroplating process. Therefore, the diffusion coefficient of 
nickel ion in this work is found to be $2.33 \times 10^{-9} \mathrm{~m}^{2} / \mathrm{s}$ by Eq. (3).

Current limit deposition $\left(\mathbf{j}_{\text {in }}<\mathbf{j}_{\text {diff }}\right)$

If the input current supply is less than the diffusion current density, the electroplating deposition rate is dominated by the input current density. The deposition rate, $\mathrm{T}$, is presented as

$T=k_{\mathrm{d}} j_{\text {in }}$

where $k_{\mathrm{d}}$ is the deposition coefficient. The height of the $n$th pixel, $h_{n}$, at time $t$ is a function of the deposition rate and the gap width, $g_{n}$, between common electrode and $n$th control electrode. The deposition time of pixel $n$ starts at $t-\mathrm{g}_{n} / T$, and the height of the $n$th pixel can be represented as:

$h_{n}=k_{\mathrm{d}} j_{\text {in }} t-g_{n}$

\section{Diffusion limit deposition $\left(\mathbf{j}_{\text {in }}=\mathbf{j}_{\text {diff }}\right)$}

When the input current supply reaches the diffusion current density, the deposition rate is determined by the diffusion of nickel ions in the diffusion limit deposition as:

$T=k_{\mathrm{d}} \frac{n F D_{0} C_{0}}{\delta}$

Experimental results show that a deposition rate of $0.27 \mu \mathrm{m} / \mathrm{min}$ is measured and $\mathrm{k}_{\mathrm{d}}$ is found to be $1.14 \times 10^{-11} \mathrm{~m}^{3} / \mathrm{C}$. The height of the $n$th pixel, $h_{n}$, at time $t$ can be described as:

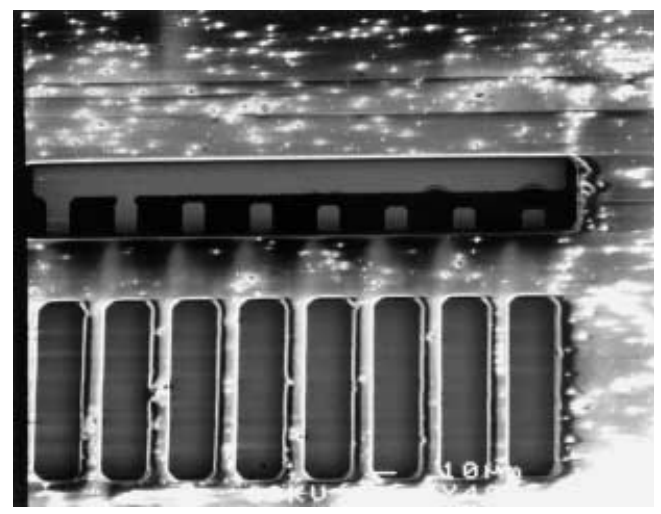

Fig. 3. The SEM microphoto shows the common electrodes and target electrodes with photoresist

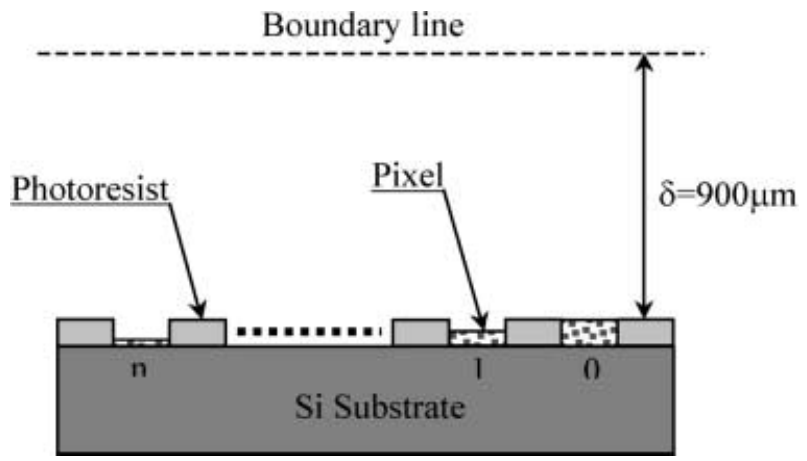

Fig. 4. The illustrative cross-sectional view showing the boundary layer $h_{n}=k_{\mathrm{d}} \frac{n F D_{0} C_{0}}{\delta} t-g_{n}$

\section{4}

\section{Experimental results and discussions}

Figure 5 is the SEM microphoto of the fabricated microstructures showing nickel deposition with $1 \mu \mathrm{m}$ increment between adjacent pixels. The highest pixel is about $9.3 \mu \mathrm{m}$ and the lowest one is $1.1 \mu \mathrm{m}$ as measured by a stylus profiler. The deposition rate is about $0.27 \mu \mathrm{m} / \mathrm{min}$ under an electroplating current density of $400 \mathrm{~A} / \mathrm{m}^{2}$. Figure 6 shows the close view SEM microphoto at the intersection area of the step-height control electrode and the common electrode. The deposition in the lateral direction can be clearly observed having equal deposition, about $4 \mu \mathrm{m}$, along both vertical and horizontal orientations on pixel number 4.

Figure 7 shows the comparison of the measured height and the developed electroplating model after $30 \mathrm{~min}$ of

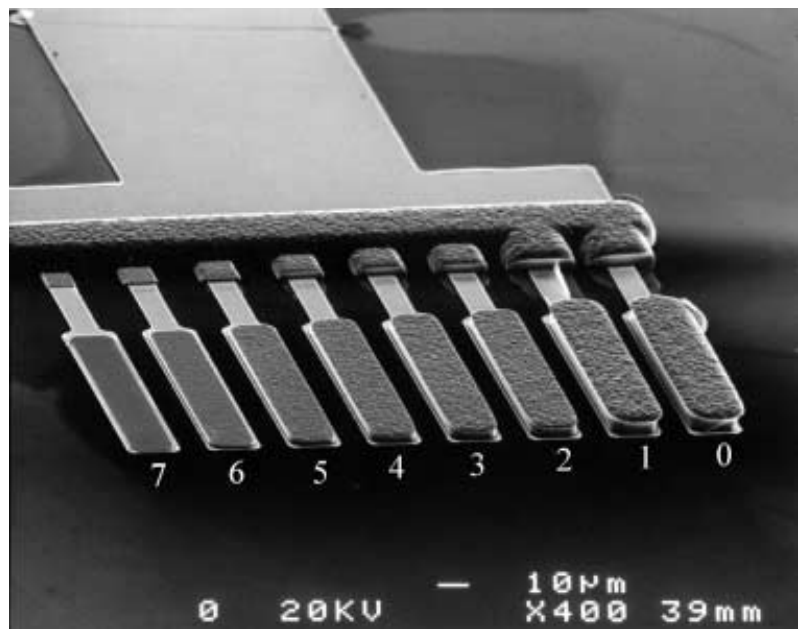

Fig. 5. The SEM microphoto showing the electroplated 3D microstructures

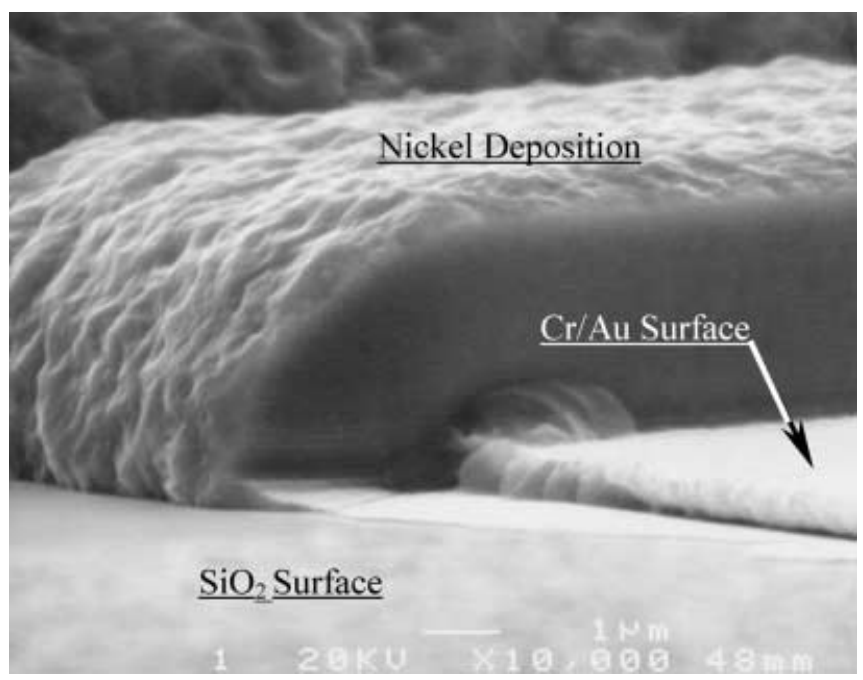

Fig. 6. The close view SEM microphoto showing at the interface of common electrode and step-height control electrode 


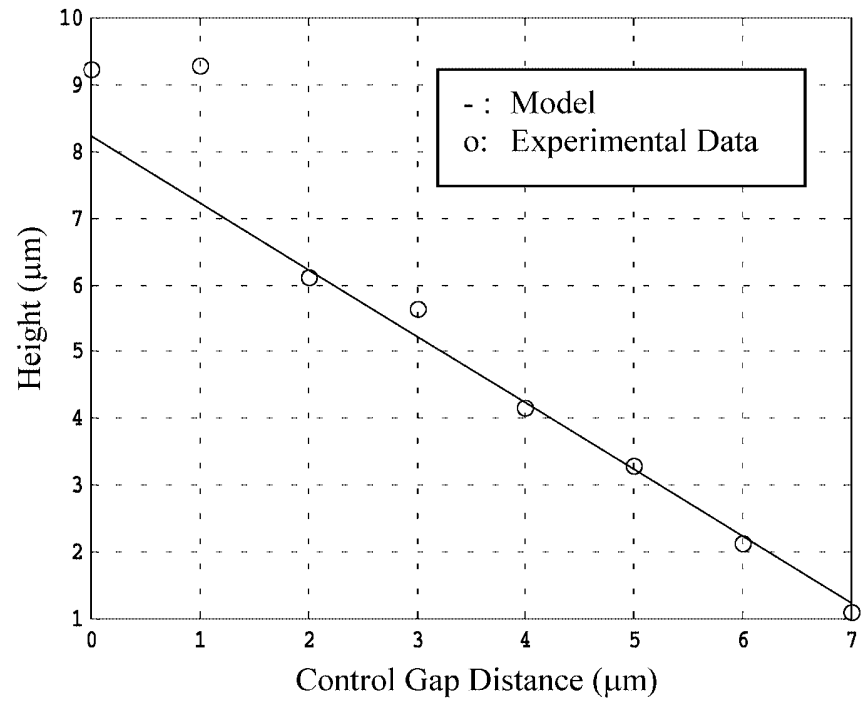

Fig. 7. The relationship of control gap distance and the deposition height

electroplating. The measured height matches the model well and the major discrepancy on pixel No. 0 and 1 is believed to be the problem of the lithography process. As shown in Fig. 3, pixel number 0 and pixel number 1 have the same height because of the poor control in the lift-off process that connected pixel number one at the beginning.

Current density fluctuation can change deposition rate after individual is activated consecutively. An open deposition area of $1 \mathrm{~cm}^{2}$ is designed to serve as a current reservoir to average out the current density fluctuation. As shown in Fig. 8, the current reservoir is designed on a separated chip such that there is no waste area on the $3 \mathrm{D}$ electroplating substrate. In the present work, this current reservoir is about 5000 time s larger than the total pixel areas such that current density fluctuation resulted by adding one pixel is only $0.002 \%$.

In the diffusion limit deposition, the electroplating deposition rate is controlled by the nickel ion diffusion flux such that the deposition rate is inversely proportional to the distance to the diffusion boundary. In this paper, the boundary is about 100 times thicker than the deposition thickness such that the linear model is sufficient. For those applications with thinner boundary or thick deposition thickness, the deposition rates of individual pixels and the lateral deposition rate in the control gaps may vary due to their different distances to the diffusion boundary.

\section{5}

\section{Conclusions}

Pixels from 9.3 to $1.1 \mu \mathrm{m}$ in height are fabricated with only two masks and one single $30 \mathrm{~min}$ electroplating process

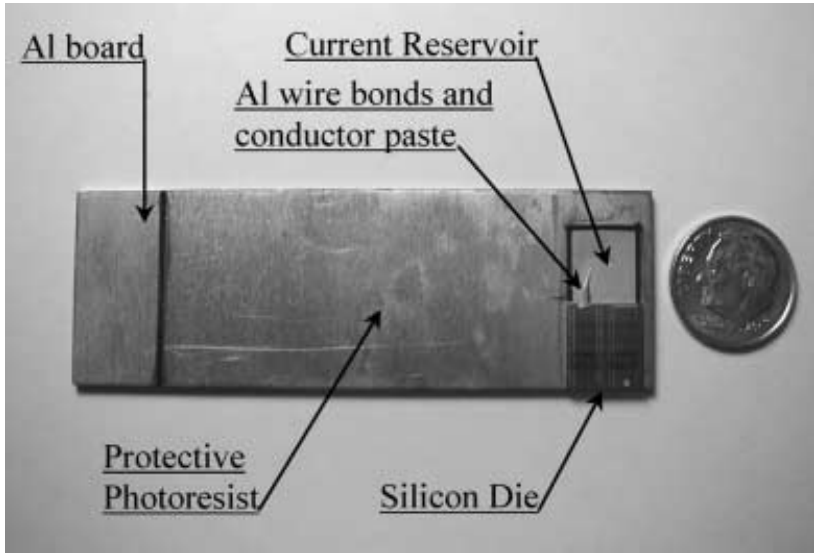

Fig. 8. The optical photo showing the current reservoir and the die

with current density of $400 \mathrm{~A} / \mathrm{m}^{2}$. Current limit and diffusion limit control models are discussed for design and prediction of the electroplating process. In this work, it is found that the diffusion constant of nickel ion is $2.33 \times 10^{-9} \mathrm{~m}^{2} / \mathrm{s}$ and the deposition coefficient is $1.14 \times 10^{-11} \mathrm{~m}^{3} / \mathrm{C}$. The demonstration of eight pixels in various heights has potential applications in the fabrication of 3D microstructures.

\section{References}

1. McCormick M et al. (1994) Microengineering design and manufacture using the LIGA process. Eng Sci Education J 3(6): 255-262

2. Cohn A et al. (1999) EFAB: low-cost automated electrochemical batch fabrication of arbitrary $3 \mathrm{D}$ microstructures. Proc SPIE, 236-247

3. Ikuta K et al. (1992) Real three dimensional micro fabrication using stereo lithography and metal molding. Proc IEEE Micro Electro Mechanical Systems, 42-47

4. Bloomstein TM et al. (1994) Laser stereo micromachining at one-half million cubic micrometers per second. Tech Digest Solid-State Sensor and Actuator Workshop, 142-144

5. Southampton Electrochemistry Group. (1985) Instrumental Methods in Electro-chemistry. Wiley, New York

6. Atkins PW (1990) Physical Chemistry. 4th edn. Freeman, New York

7. Bard AJ; Faulkner R (1980) Electrochemical Methods: Fundamentals and Applications. Wiley, New York

8. White FM (1986) Fluid Mechanics. 2nd edn. McGraw-Hill, New York

9. Madden JD; Hunter IW (1996) Three-dimensional microfabrication by localized electrochemical deposition. J Microelectromech Syst 5(1)

10. MacDermid, Inc., 245 Freight Street, Waterbury, CT 06702 